\title{
SOLUTIONS OF SOME PERIODIC STIELTJES INTEGRAL EQUATIONS
}

\author{
G. W. MARRAH AND T. G. PROCTOR
}

\begin{abstract}
Nonlinear periodic perturbations of a family of linear periodic Stieltjes integral equations are considered and sufficient conditions are given for the existence of a periodic solution for one member of the family. Conditions are given under which the solutions of the family approach the periodic solution asymptotically. A Floquet type theorem for periodic Stieltjes integral equations and several examples are given.
\end{abstract}

Let $S$ be an interval of numbers of the form $[\tau, \infty)$, let $\{G,|\cdot|\}$ be a complete normed abelian group and denote the set of all functions from $G$ to $G$ by $H$. A function $V$ from $S \times S$ into $H$ such that $V(x, y)+V(y, z)=$ $V(x, z)$ whenever $y$ is between $x$ and $z$ will be called an order additive function. Suppose that each of $V_{1}$ and $V_{2}$ is an order additive function such that

(1) $V_{1}(x, y) 0=0$ for all $(x, y)$ in $S \times S$ where 0 is the zero element of $G$;

(2) there are order additive functions $\alpha_{1}, \alpha_{2}$ from $S \times S$ to the nonnegative numbers such that

$$
\left|V_{i}(x, y) p-V_{i}(x, y) q\right| \leqq \alpha_{2}(x, y)|p-q|, \quad\left|V_{2}(x, y) 0\right| \leqq \alpha_{2}(x, y),
$$

for each $(x, y)$ in $S \times S,(p, q)$ in $G \times G$ and $i=1,2$. We will also suppose that $V_{2}(x, y) 0 \neq 0$ for some $(x, y)$ in $S \times S$.

The first result in this paper is a Floquet theorem which says that under periodicity conditions on $V_{1}$, a knowledge of the solution of

$$
h(t)=p+(R) \int_{t}^{t} V_{1} h
$$

over a period $\tau \leqq t \leqq \tau+\omega$ gives the values for all $\tau \leqq t$. The second theorem describes conditions under which there is a $p$ in $G$ such that the solution of

$$
h(t)=p+(R) \int_{t}^{\tau}\left(V_{1}+V_{2}\right) h
$$

is periodic. Mac Nerney gives the existence and uniqueness theorems for

Received by the editors August 10, 1971 and, in revised form, October 27, 1971.

AMS 1969 subject classifications. Primary 4513; Secondary 3453, 3445.

Key words and phrases. Stieltjes integral equations, order additive, Floquet theorem, periodic, difference equation, perturbation of linear ordinary differential equation.

(c) American Mathematical Society 1972 
equations (1) and (2) in [3]. The second result generalizes a well-known theorem concerning the existence of a periodic solution of a perturbation of a noncritical system of linear differential equations. Here a variation of parameter theorem obtained by Reneke [6] is the primary tool used. Theorem 3 gives conditions under which the solution obtained from Theorem 2 is stable.

Let $K$ map $S \times S$ into $H$ and have the property that there is a positive number $\omega$ such that $K(x, y)=K(x+\omega, y+\omega)$ for all $(x, y)$ in $S \times S$; then we will say that $K$ has the $\omega$ period property.

REMARK. If $K$ is order additive it is easy to see that $K$ has the $\omega$ period property if and only if $\omega$ is a positive number such that

$$
K(x, x+\omega)=K(y, y+\omega) \text { and } K(x+\omega, x)=K(y+\omega, y) .
$$

Mac Nerney [3] shows that there is a function $W_{1}$ from $S \times S$ into $H$ defined by

$$
W_{1}(x, y) p={ }_{x} \prod^{y}\left(I+V_{1}\right) p
$$

for $(x, y)$ in $S \times S$ and $p$ in $G$, that $W_{1}$ is order multiplicative (i.e. $W_{1}(x, y) W_{1}(y, z)=W_{1}(x, z)$ for all $y$ between $x$ and $\left.z\right)$, and that $h$ given by $h(t)=W_{1}(t, \tau) p$ is the unique solution of (1). Here $I$ is the identity map on G.

LEMMA 1. If $V_{1}$ has the $\omega$ period property then $W_{1}$ has the $\omega$ period property.

The proof follows from the definition of $W_{1}$ and a simple translation of partitions of the interval $x$ to $y$ into the interval $x+\omega$ to $y+\omega$.

THEOREM 1. If $V_{1}$ has the $\omega$ period property, $n$ is a positive integer and $t \geqq \tau$ then

$$
W_{1}(t+n \omega, \tau)=W_{1}(t, \tau) W_{1}(\tau+\omega, \tau)^{n} .
$$

The proof follows by induction using the order multiplicative property of $W_{1}$.

REMARK 1. Under the hypothesis of Theorem 1 , if $W_{1}(\tau+t, \tau)$ is known for $0 \leqq t \leqq \omega$ then the solution $h$ of (1) is known for all $t \geqq \tau$.

REMARK 2. If $W(\tau+\omega, \tau)=I$ then for any $p$ in $G$ the solution of (1) is periodic (i.e. $h(t+\omega)=h(t)$ for $t \geqq \tau$ ).

REMARK 3. Let $A$ be a continuous $n \times n$ matrix valued function from $S$ with period $\omega$, let $V_{1}(x, y) p=-\left[\int_{x}^{y} A(s) d s\right] p$ for $p$ in $R^{n}$ and let $h_{0}$ be a continuous function from $[\tau, \infty)$ into $R^{n}$, then

$$
\text { (R) } \int_{t}^{\tau} V h_{0}=\int_{\tau}^{t} A(s) h_{0}(s) d s .
$$


Consequently, since the sequence $h_{n}(t)$ given by $h_{n}(t)=p+(R) \int_{t}^{\tau} V h_{n-1}$, $n=1,2, \cdots$, converges uniformly to the solution of (1) (see Theorem 2 in [3]) we have $W_{1}(t, \tau) p=X(t) X^{-1}(\tau) p$ where $X$ is a fundamental matrix of solutions of $x^{\prime}=A(t) x$. Theorem 1 in this case is the usual Floquet theorem (see Hale [1]).

REMARK 4 . Let $S=[0, \infty)$, let $A$ be an $n \times n$ matrix valued function from $S$ which has local bounded variation and let ( ): $S \rightarrow R$ be given by $(t)=n-1$ for $n-1<t \leqq n$ where $n$ is a positive integer. The solution of the vector difference equation $y(n+1)=A(n) y(n)$ satisfies

$$
y(n)=y(0)+(R) \int_{n}^{0} V y,
$$

where $V(u, v) y=-(R) \int_{u}^{v}\left(A-I^{*}\right) d() \cdot y$ and conversely. Here $I^{*}$ is the $n \times n$ identity matrix. Consequently, Theorem 1 is a Floquet theorem for difference equations.

REMARK 5. If $V_{2}(x, y) 0 \neq 0$ for all $(x, y)$ in $S \times S$ we can establish similar results for an integral equation of the form

$$
h(t)=p+(R) \int_{t}^{r} V_{2} h .
$$

Following Mac Nerney [3], let $G^{*}=\{(g, m): g \in G, m \in\{0,1\}\}$, let addition in $G^{*}$ be componentwise and addition in the second component be modulo 2 and let $|(g, m)|=|g|+m$. It is easy to verify that $\left(G^{*},|\cdot|\right)$ is a complete normed abelian group. Let $V^{*}$ be a map from $S \times S$ to $H^{*}$ given by

$$
V^{*}(x, y)(g, m)=\left(V_{2}(x, y) p-V_{2}(x, y) 0+m V_{2}(x, y) 0,0\right) .
$$

We note that $V^{*}(x, y) 0=0$ and that if $V_{.2}$ has the $\omega$ period property so does $V^{*}$. It is easy to see that for $W^{*}: S \times S \rightarrow H^{*}$ given by

$$
W^{*}(x, y)(g, m)={ }_{x} \prod^{y}\left(I+V^{*}\right)(g, m)
$$

and $W_{3}: S \times S \rightarrow H$ given by

$$
W^{*}(x, y)(g, 1)=\left(W_{3}(x, y)(g, 1), 1\right)
$$

the function $h=W_{3}(, \tau)(p, 1)$ is the solution of (4). Consequently if $W^{*}(\tau+t, \tau)$ is known for $0 \leqq t \leqq \omega$ the solution of (4) is known for all $t \geqq \tau$.

For the remaining portion of the paper let $V_{1}(x, y)$ be a group homomorphism of $G$ for each $(x, y)$ in $S \times S$. It is easy to see that $W_{1}$ defined by (3) also has its values in the group homomorphisms of $G$. Let $\omega$ be a positive number and QC be the collection of quasi-continuous functions $h$ 
from $[\tau, \tau+\omega]$ to $G$ (i.e., $h\left(t^{+}\right)$exists for $\tau \leqq t<\tau+\omega$ in $S, h\left(t^{-}\right)$exists for $\tau<t \leqq \tau+\omega)$ with $h(\tau)=h(\tau+\omega)$, and for $h$ in QC let

$$
\|h\|=\sup _{r \leqq t \leqq \tau+\omega}|h(t)| .
$$

LEMMA 2. For $h$ in $Q C$ the function $k$ given by

$$
k(t)=(L, R) \int_{t}^{\tau} W_{1}(t,) V_{2} h, \quad \tau \leqq t \leqq \tau+\omega,
$$

exists and has bounded variation.

The proof is similar to the proof of Lemma 2.2 in Mac Nerney [3] and thus will not be given here.

Mac Nerney [3] also shows that the function $\mu_{1}: S \times S$ to the numbers not less than 1 given by

$$
\mu_{1}(x, y)={ }_{x} \Pi^{v}\left(1+\alpha_{1}\right)
$$

exists and $\left|W_{1}(x, y) p\right| \leqq \mu_{1}(x, y)|p|$.

THEOREM 2. If $V_{1}, V_{2}$ have the $\omega$ period property, if the map $I-W_{1}(\omega+\tau, \tau)$ is reversible and there is an $M>0$ such that

$$
\left|\left[I-W_{1}(\omega+\tau, \tau)\right]^{-1} g\right| \leqq M|g| \text { for } g \in G,
$$

and if

$$
(L) \int_{\tau+\omega}^{\tau} \mu_{1}(\tau+\omega, \quad) \alpha_{2}<\frac{1}{\mu_{1}(\tau+\omega, \tau) M+1},
$$

then there is a unique $p$ in $G$ such that the solution $h$ of (2) has period $\omega$ (i.e. $h(t+\omega)=h(t)$ for $t \geqq \tau)$.

Proof. For $f$ in QC define

and

$$
P_{f}=\left[I-W_{1}(\tau+\omega, \tau)\right]^{-1}(L, R) \int_{\tau+\omega}^{\tau} W_{1}(\tau+\omega, \quad) V_{2} f
$$

$$
T f(t)=W_{1}(t, \tau) P_{f}+(L, R) \int_{t}^{\tau} W_{1}(t,) V_{2} f .
$$

We note that since

$$
\begin{array}{rl}
T f(\tau)-T & f(\tau+\omega) \\
& =\left[I-W_{1}(\tau+\omega, \tau)\right] P_{f}-(L, R) \int_{\tau+\omega}^{\tau} W_{1}(\tau+\omega, \quad) V_{2} f=0,
\end{array}
$$

$T$ maps QC into itself $\left(W_{1}(, \tau)\right.$ has bounded variation, see Mac Nerney 
[3]). Also for $f, h$ in QC we have

and similarly

$$
\begin{aligned}
\left|P_{f}-P_{h}\right| & \leqq M\left|(L, R) \int_{\tau+\omega}^{\tau} W_{1}(\tau+\omega, \quad)\left[V_{2} f-V_{2} h\right]\right|, \\
& \leqq M(L) \int_{\tau+\omega}^{\tau} \mu_{1}(\tau+\omega, \quad) \alpha_{2}\|f-h\| ;
\end{aligned}
$$

$$
|T f(t)-T h(t)| \leqq\|f-h\|\left[M \mu_{1}(\tau+\omega, \tau)+1\right](L) \int_{\tau+\omega}^{\tau} \mu_{1}(\tau+\omega, \quad) \alpha_{2} .
$$

Consequently by the Contraction Mapping Theorem there is an $f$ in QC such that

(5) $\quad f(t)=W_{1}(t, \tau) P_{f}+(L, R) \int_{t}^{\tau} W_{1}(t, \quad) V_{2} f, \quad \tau \leqq t \leqq \tau+\omega$.

Let $h$ be the function defined on $\tau \leqq t$ into $G$ by $h(t)=f(t-n \omega)$ where $n$ is the positive integer such that $\tau+n \omega \leqq t<(n+1) \omega+\tau$. Clearly $h$ is quasi-continuous and periodic and

$$
\begin{aligned}
h(t)= & W_{1}(t-n \omega, \tau) P_{f}+(L, R) \int_{t-n \omega}^{\tau} W_{1}(t-n \omega, \quad) V_{2} f \\
= & W_{1}(t, \tau+n \omega) P_{f}+(L, R) \int_{t}^{\tau+n \omega} W_{1}(t, \quad) V_{2} h \\
= & W_{1}(t, \tau+n \omega)\left[W_{1}(\tau+n \omega, \tau) P_{f}+(L, R) \int_{\tau+n \omega}^{\tau} W_{1}(n \omega+\tau,) V_{2} h\right] \\
& +(L, R) \int_{t}^{\tau+n \omega} W_{1}(t,) V_{2} h ;
\end{aligned}
$$

thus

$$
h(t)=W_{1}(t, \tau) P_{f}+(L, R) \int_{t}^{\tau} \dot{W}_{1}(t, \quad) V_{2} h, \quad \tau \leqq t .
$$

Reneke [6] has shown that $h$ is a solution of (6) if and only if $h$ is a solution of (2) with $p$ replaced by $P_{f}$.

For the uniqueness part of the theorem we note that if $h^{*}$ is a solution of (2) with period $\omega$ then by Reneke's result $T h^{*}=h^{*}$. Since $T$ has exactly one fixed point $h=h^{*}$ and thus $p=P_{f}$.

REMARK 6. Take $V_{1}$ as in Remark 3 and let $V_{2}$ be given by

$$
V_{2}(x, y) p=-\int_{x}^{y} f(s, p) d s, \quad(x, y) \text { in } S \times S, \quad p \text { in } G,
$$

where $f$ is a continuous function from $S \times R^{n}$ into $R^{n}$ with $f(t+\omega, p)=$ $f(t, p)$ and $|f(t, p)-f(t, q)| \leqq L|p-q|$. The solution of (2) satisfies

$$
x^{\prime}=A(t) x+f(t, x)
$$


thus Theorem 2 generalizes a well-known theorem in ordinary differential equations (see Hale [1, Theorem 5-1]).

For any $f$ in $H$ satisfying a Lipschitz condition and $f(0)=0$ let

$$
N(f)=\sup \{|f(p)| /|p|: p \in G, p \neq 0\} .
$$

In the case $G$ is a Banach space Martin has shown [5] that the functions $\sigma$ and $\pi$ on $S \times S$ given by

$$
\sigma(a, b)={ }_{a} \sum^{b}\left[N\left(I+V_{1}\right)-1\right], \quad \pi(a, b)={ }_{a} \prod^{b} N\left(W_{1}\right)
$$

exists, $\left|W_{1}(t, \tau) p\right| \leqq \pi(t, \tau)|p|, \sigma$ is order additive and for any real number $\delta$, the unique solution of the integral equation $\chi(t)=\delta+(R) \int_{t}^{\tau} \sigma \chi$ is given by $\chi(t)=\pi(t, \tau) \delta$.

REMARK 7. In the case that $G$ is a Banach space, condition (b) in Theorem 2 may be replaced by

$$
\sup _{\tau \leqq t \leqq \tau+\omega}\left\{\pi(t, \tau) M(L) \int_{\tau+\omega}^{\tau} \pi(\tau+\omega,) \alpha_{2}+(L) \int_{t}^{\tau} \pi(t, \quad) \alpha_{2}\right\}<1
$$

and the conclusion of the theorem follows using the same proof.

THEOREM 3. Let $G$ be a Banach space and let the hypothesis of Theorem 2 hold. Further suppose that for any number $\delta$, the solutions $k_{\delta}$ of

$$
k(t)=\delta+(R) \int_{t}^{\tau}\left(\sigma+\alpha_{2}\right) k
$$

satisfy $\lim _{t \rightarrow \infty} k_{\delta}(t)=0$ where $\sigma$ is defined above. If $h$ is the periodic solution of Theorem 2 and $h^{*}$ is the solution of (2) with $h^{*}(\tau)=p^{*}$,

$$
\lim _{t \rightarrow \infty}\left[h^{*}(t)-h(t)\right]=0 .
$$

Proof. According to the variation of parameters formula devised by Reneke [6],

$$
h^{*}(t)=W_{1}(t, \tau) p^{*}+(L, R) \int_{t}^{\tau} W_{1}(t, \quad) V_{2} h^{*}
$$

and $h$ satisfies a similar equation. Thus for $\lambda(t)=\left|h^{*}(t)-h(t)\right|$ we have

$$
\lambda(t) \leqq \pi(t, \tau)\left|p-p^{*}\right|+(L, R) \int_{t}^{\tau} \pi(t, \quad) \alpha_{2} \lambda .
$$

The function $k_{\delta}$ where $\delta=\left|p-p^{*}\right|$ satisfies

$$
k_{\delta}(t)=\pi(t, \tau)\left|p-p^{*}\right|+(L, R) \int_{t}^{\tau} \pi(t, \quad) \alpha_{2} k_{\delta}
$$

and by a Gronwall type result given by Marrah [4] we have $\lambda(t) \leqq k_{\delta}(t)$. 
REMARK 8. For the $V_{1}, V_{2}$ specified in Remark 6,

$$
\begin{aligned}
\sigma(a, b) & =\int_{a}^{b} \mu[A(s)] d s & & \text { if } a \leqq b, \\
& =-\int_{a}^{b} \mu[A(s)] d s & & \text { if } a>b,
\end{aligned}
$$

where $\mu$ is the logarithmic norm

$$
\mu[A(s)]=\lim _{h \rightarrow 0^{+}}(|I+h A(s)|-1) / h ;
$$

consequently, the solution of equation (7) satisfies

$$
d k / d t=(\mu[A(t)]+L) k .
$$

In this case Theorem 3 is a restatement of a known result that if $\mu[A(s)]+L<0$ then the periodic solution furnished by Theorem 2 is asymptotically stable.

\section{REFERENCES}

1. J. K. Hale, Oscillations in nonlinear systems, McGraw-Hill, New York, 1963. MR 27 \#401.

2. - Functional differential equations, Springer-Verlag, New York, 1971.

3. J. S. Mac Nerney, A nonlinear integral operation, Illinois J. Math. 8 (1964), 621-638. MR 29 \#5082.

4. G. W. Marrah, Qualitative theory for Stieltjes integral equations, Ph.D. Dissertation, Clemson University, Clemson, S.C., 1971.

5. R. H. Martin, Jr., Bounds for solutions to a class of nonlinear integral equations, Trans. Amer. Math. Soc. 160 (1971), 131-138.

6. J. A. Reneke, $A$ variation of parameter formula, Clemson Mathematics Department Report \#87, Clemson, S.C., 1971.

Department of Mathematics, Virginia Military Institute, Lexington, Virginia 24450

Mathematical Sciences Department, Clemson University, Clemson, South CAROLINA 29631 